\title{
Meta-analysis of association between TCF7L2 rs7903146 and risk of new-onset diabetes after transplantation
}

\author{
Muhammad Tassaduq Khan
}

Renal Transplant Unit, Dow University Hospital, Karachi, Pakistan

Background: Single nucleotide polymorphisms may influence the risk of development of new-onset diabetes after transplantation (NODAT), a post-transplant clinical complication that is often implicated in allograft rejection and mortality. We performed a meta-analysis of association between TCF7L2 rs7903146 and risk of post-transplant diabetes mellitus.

Methods: A systematic search was conducted using PubMed and ScienceDirect electronic databases for studies published between January 2001 to January 2021. Case-control or cohort studies reporting association between NODAT (diagnosis based on American Diabetes Association criteria) and TCF7L2 rs7903146 were included. MetaGenyo was used for meta-analysis (random effects model). Pooled odds ratios with $95 \%$ confidence intervals were reported to evaluate the strengths of association.

Results: Two reviewers independently screened for articles. A total of six case-control studies were included for full-text review and quantitative analysis after screening for eligibility. Genotypic distributions were in Hardy-Weinberg equilibrium for included studies. All papers reported statistically significant association of TCF7L2 rs7903146 for risk of NODAT, except for one study. There was moderate heterogeneity among studies $\left(I^{2}=60.6 \%\right)$. Pooled analysis revealed $51 \%$ odds of developing NODAT with TCF7L2 rs7903146 T allele (allele contrast model: odds ratio, 1.51; 95\% confidence interval, 1.13-2.02; adjusted $\mathrm{P}=0.03$ ).

Conclusions: The present meta-analysis demonstrated association between TCF7L2 variant rs7903146 and risk of developing NODAT. This finding may have clinical implications for individuals undergoing kidney transplantation.

Corresponding author: Muhammad Tassaduq Khan

E-mail: Muhammad.tassaduq@duhs.edu.pk

(c) The Korean Society for Transplantation

This is an Open Access article distributed under the terms of the Creative Commons Attribution Non-Commercial License (http://creativecommons.org/licenses/by-nc/4.0/) which permits unrestricted non-commercial use, distribution, and reproduction in any medium, provided the original work is properly cited. 